\title{
Tangence
}

\section{L'actualité de la littérature acadienne}

\section{Raoul Boudreau}

Numéro 58, octobre 1998

Le postmoderne acadien

URI : https://id.erudit.org/iderudit/025976ar

DOI : https://doi.org/10.7202/025976ar

Aller au sommaire du numéro

Éditeur(s)

Tangence

ISSN

0226-9554 (imprimé)

1710-0305 (numérique)

Découvrir la revue

Citer cet article

Boudreau, R. (1998). L'actualité de la littérature acadienne. Tangence, (58), 8-18. https://doi.org/10.7202/025976ar d'utilisation que vous pouvez consulter en ligne.

https://apropos.erudit.org/fr/usagers/politique-dutilisation/ 


\section{L'actualité de la littérature acadienne Raoul Boudreau}

L'année qui se termine marque certes un jalon pour la littérature acadienne, celui du vingt-cinquième anniversaire de la fondation des Éditions d'Acadie, c'est-à-dire de l'éclosion de la littérature acadienne telle que nous la connaissons aujourd'hui. Sans le moindrement minimiser le travail de pionnier des patients historiens de la littérature acadienne qui font remonter ses origines aussi loin que la colonie elle-même, on ne peut nier que 1972 constitue un saut qualitatif, le dépassement d'un seuil au-delà duquel le mot "littérature" n'a plus le même sens parce qu'elle s'appuie sur une structure plus élaborée et plus solide, qu'elle mérite désormais d'être appelée une "institution".

Le moment est donc propice, sinon à l'établissement d'un bilan qui apparaîtrait par trop hâtif, du moins à une prise de distance critique, à une tentative de cerner ses forces et ses faiblesses, d'établir certaines de ses orientations, l'originalité de sa situation, qui peut justifier l'intérêt des littératures voisines ou parentes.

Quoi qu'en pensent les écrivains acadiens qui ont choisi de résister à l'appel du Québec, il n'en reste pas moins que celui-ci constitue pour la littérature acadienne une incontournable pierre de touche. C'est encore, pour plusieurs, à l'aune de la réussite au Québec que se mesure l'importance de l'écrivain acadien et, devant l'envergure et les moyens de l'institution littéraire québécoise, l'équivalent acadien ne fait pas le poids. Antonine Maillet, Jacques Savoie et Serge Patrice Thibodeau sont autant de preuves que pour dépasser un certain seuil de diffusion et de reconnaissance, l'écrivain acadien doit se faire québécois. Le Québec occupe donc par rapport à l'écrivain acadien une position des plus ambiguë: il est d'un certain côté le terrain sur lequel il aspire à se faire reconnaître et d'un autre cồté la force dominatrice dans laquelle il doit aliéner une partie de lui-même pour accéder à cette reconnaissance.

La littérature acadienne est à la périphérie d'une sphère dont le Québec est le centre. François Paré rappelle que l'université 
québécoise à partir de la fin des années soixante a réussi à transformer l'image de la langue et de la littérature québécoise dans la tentative d'en faire une littérature dominante. Et il poursuit:

$\mathrm{Et}$, en fait le stratagème a réussi au delà de toutes les attentes, puisqu'à partir de 1970 environ les lettres québécoises disposent de toutes les structures institutionnelles nécessaires et se proposent donc dans le concert des littératures mondiales comme une littérature hégémonique. C'est là un renversement idéologique absolument extraordinaire qui a modifié profondément, non seulement les rapports entre la France et le Québec, mais aussi et surtout ceux que le Québec entretenait et entretient encore avec les autres littératures de l'exiguité. ${ }^{1}$

On ne s'étonnera donc pas du peu d'intérêt manifesté au Québec pour la littérature acadienne. (Ce numéro de la revue Tangence est une heureuse et rare exception à cette tendance.) Pour la majorité des Québécois, la littérature acadienne reste attachée, en raison de sa diffusion massive par la télévision, à la figure de la Sagouine, son accent rocailleux, ses mots de l'empremier, mais surtout ce personnage est le signe d'une société tournée vers le passé et il incarne à merveille ce dont le Québec doit se distancer pour se doter d'une littérature dominante, c'est-à-dire l'oralité et ses connotations régionalistes. La vocation de la littérature acadienne est ainsi toute trouvée. Sans nier cette vision de l'Acadie qui permet au Québec de s'en servir comme repoussoir, il importe de montrer une autre production littéraire qui témoigne de préoccupations qui rejoignent celles de nombreux écrivains contemporains de tous horizons.

\section{La poésie d'abord}

Ce que la majorité des Québécois ignorent, c'est que malgré tout le mérite de l'œuvre d'Antonine Maillet, dont le Goncourt n'est pas le moindre, elle est peut-être l'arbre qui cache la forêt. Pendant qu'elle remportait le succès que l'on sait au Québec et en France, en réussissant à faire croire à ces origines retrouvées, à une langue miraculeusement conservée, plus française que l'hexagonale, commencèrent à écrire et à publier, tout un groupe de jeunes poètes, bien davantage poussée par une nécessité de

1 François Paré, Les littératures de l'exiguité, Hearst, Le Nordir, 1992, p. 42. (C'est nous qui soulignons.) 
s'exprimer que par le désir de faire de la littérature ${ }^{2}$, dont ils n'avaient pour la plupart qu'une idée assez vague et fort heureusement pour eux, car l'envie leur serait-elle venue d'aller voir ce qu'était la littérature, ils se seraient sans doute arrêtés d'écrire, en constatant comme ils étaient mal armés, du point de vue de la maîtrise de la langue, pour jamais y accéder.

Comme dans la plupart des littératures émergentes, ces poètes ont d'abord été obsédés par l'identité, la revendication nationalitaire et il est bien évident que les poètes québécois du pays ont été pour eux des modèles privilégiés ${ }^{3}$. De cette période du début des années soixante-dix, tout n'est pas effacé et il subsiste des recueils, Cri de terre ${ }^{4}$, Acadie Rock ${ }^{5}$, Mourir à Scoudouc ${ }^{6}$, dont la valeur littéraire a transcendé les impératifs idéologiques ponctuels.

Ce qui est peut-être plus étonnant et même déroutant pour un regard extérieur, c'est que la poésie acadienne a assez vite tourné le dos à cette veine nationaliste et régionaliste. La défense d'un néo-nationalisme s'opposant à l'ancien par son côté revendicateur et révolté est certes le thème privilégié de la première vague de la poésie acadienne. Au cours des années 80, la poésie acadienne change de ton, en grande partie sous l'influence des femmes-poètes qui accèdent en même temps à l'écriture et jouent à ce moment-là les premiers rôles en poésie acadienne, position de force qu'elles n'occupent plus aujourd'hui, la relève se faisant beaucoup plus du côté masculin. François Paré montre de manière convaincante le rôle des femmes dans l'éclatement thématique et formel de la poésie acadienne? ${ }^{7}$ Cependant je n'affirmerais pas de manière aussi absolue que "le devenir [de l'Acadie] constitue toujours [...] le cour du discours poétique acadien dans son ensemble" 8 . C'est à mon avis un aspect des choses, mais il

2 On trouvera le meilleur compte rendu de cette naissance dans Lectures acadiennes d'Alain Masson, Moncton, Éditions Perce-Neige, l'Orange bleue éditeur, 1994.

3 Voir mon article "La poésie acadienne et le Québec", Acadiensis, printemps 1992, p. 139-145.

4 Raymond Guy LeBlanc, Éditions d'Acadie, 1972, nouvelle éditions 1992.

5 Guy Arsenault, Éditions d'Acadie, 1973, $2^{2}$ édition Éditions Perce-Neige/Écrits des Forges, 1994.

6 Herménégilde Chiasson, Éditions d'Acadie, 1974.

7 François Paré, . La chatte et la toupie: écriture féminine et communauté en Acadie •, Francopbonies d'Amérique, no 7, 1997, p. 115-126.

8 Ibid., p. 116. 
est contrebalancé par la tendance opposée, peut-être tout aussi révélatrice de cette obsession par son refoulement, c'est-à-dire la fuite dans le fantastique et le rêve, la dénonciation et la déconstruction de la pensée patriotarde, la revendication de destins individuels où l'écriture occupe la première place et ne saurait être asservie à d'autres causes qu'elle-même.

Certes, le passé, le présent et le devenir de l'Acadie hantent la poésie d'Herménégilde Chiasson, et de manière particulièrement prégnante et grave dans son dernier recueil ${ }^{9}$. Mais ce n'est certes pas une thématique privilégiée chez Serge Patrice Thibodeau qui en fait le sujet d'un unique recueil sur les sept publiés depuis 1990. Ce n'est pas davantage le cas dans les quatre recueils de Fredric Gary Comeau ou dans les deux recueils publiés par Mario Thériault, pas plus que chez Marc Arseneau et encore moins chez Maurice Raymond ${ }^{10}$, et on pourrait poursuivre cette énumération. En définitive, $H$. Chiasson et G. Leblanc sont les seuls dont on puisse dire que l'Acadie a toujours eu une forte présence dans leur poésie, dont l'écriture a en quelque sorte partie liée avec le destin collectif, dans le sens où la quête personnelle se confond avec la quête collective. Mais Gérald Leblanc ne partage aucunement la gravité de son complice et il est comme un poisson clans l'eau dans l'Acadie bilingue et même plurilingue du sud-est; ses poèmes ne communiquent pas la menace ou la crainte d'être avalé par l'Autre, mais la jouissance, la musique et la danse de cette fête contemporaine où l'Autre enrichit la diversité ambiante à laquelle tous sont conviés. Chiasson reste un transfuge de l'unilingue Péninsule acadienne et il n'aborde pas avec la même naïveté la mobilité linguistique de Moncton. Certes le deuxième recueil d'Ulysse Landry est une suite du premier publié en 1975 et il en conserve la thématique, mais c'est là une lacune qui relève davantage de la difficulté de se renouveler que de la persistance d'une vision. De la même manière, on trouvera chez Marc Poirier une imitation sans doute inconsciente poussée presque à la caricature du Gérald Leblanc des années soixantedix. Ce qui est indéniable cependant, c'est que la poésie acadienne, sauf rare exception comme chez Serge Patrice Thibodeau

9 Climats, Éditions d'Acadie, 1996.

10 En dehors des écrivains acadiens absorbés par la littérature québécoise, Antonine Maillet, Jacques Savoie, Serge Patrice Thibodeau, tous les autres publient la très grande majorité de leurs livies aux Éditions d'Acadie et alux Éditions Perce-Neige. 
12

et Maurice Raymond, porte, de toutes les manières possibles et surtout dans sa langue, les traces de son lieu d'énonciation.

La poésie acadienne dans son ensemble me semble caractérisée par la quête individuelle, l'expérience moderne du vide de l'existence et des mots. Si l'on y ressent très fortement le sentiment de la précarité de l'être et de son étrangeté au monde, il n'est pas dit que cette impossibilité de vivre soit principalement liée au destin collectif acadien; même si celui-ci l'aiguise, elle s'exprime dans une ouverture et une extension qui permettent au poète acadien de dépasser la marginalité où l'histoire l'a enfermé. En ce sens, l'expérience authentique de l'écriture ne peut jamais être totalement un repli sur soi.

C'est Herménégilde Chiasson qui, me semble-t-il, réussit le mieux la jonction entre la particularité acadienne et le sentiment moderne et universel d'échec et de désillusion en faisant osciller son regard au gré des recueils successifs et à l'intérieur de ceux-ci de l'un à l'autre de ces pôles. Si Vermeer, toutes les photos du film (1995) n'affiche pas aussi ouvertement les préoccupations collectives, elles sont déjà plus présentes dans Miniatures (1996), et Climats vibre de cette lancinante plainte, du cri de l'impossibilité de vivre, de la désolation irréductible, d'une agonie qui n'en finirait pas de durer et qui s'incarne profondément dans une réalité d'ici, ce qui explique sans doute la charge émotive remarquable du recueil.

Si la poésie acadienne peut offrir quelque intérêt aujourd'hui, ce ne peut être qu'en raison de sa situation particulière et paradoxale. Certes les poètes acadiens des années soixante-dix ne pouvaient s'appuyer sur une tradition littéraire véritablement constituée et ils traînaient le lourd héritage d'une langue stigmatisée, quasihonteuse et d'un sentiment d'extraterritorialité collé à la peau. Cette poésie a pu quand même prendre son envol en raison, pour une bonne part, de la conjoncture favorable créée par la montée du postmodernisme et la perte de confiance dans les grands récits, notamment dans le discours nommé Littérature et la valorisation corollaire des discours marginaux, minoritaires et hétérogènes. Émergeant au coeur d'une vaste crise de langage, la poésie acadienne trouvait spontanément à faire servir sa propre expérience d'un langage inadéquat. C'est certes un paradoxe de trouver dans la poésie acadienne si jeune les échos d'un semblable sentiment de méfiance envers le langage que celui qu'expriment des traditions poétiques au terme d'un très long cheminement. Si l'on peut désigner l'une et les autres du même qualificatif de "postmoderne" 
dans la mesure où celui-ci en est venu à nommer toute production culturelle contemporaine, on voit bien qu'il s'agit d'univers irréductibles et que la régression linguistique y fonctionne de manière opposée. Depuis Mallarmé, l'écriture est "traversée du néant" et "vit de son pouvoir de mise à distance du réel qui n'existe qu'absent" ${ }^{11}$ comme l'écrit Jean-Michel Rabaté. La poésie contemporaine construit son espace paradoxal aux confins du silence, du vide et de l'absence et la poésie acadienne transmet aussi le sentiment de son absolue précarité par l'interrogation constante sur la langue qui la porte et qui la perd. Mais l'aliénation linguistique dont elle témoigne est plus primitive que sophistiquée, plus fruste que subtile, moins nostalgique d'une plénitude passée et plus naïve. Ces vers de Fredric Gary Comeau, si ce n'était de leur musique, qu'on pourrait qualifier ironiquement de "rocailleuse", ne sont pas si loin de l'inspiration d'un André Du Bouchet:

il me restera toujours cette soif

d'avancer vers vos yeux d'absence

un langage impossible à décrypter

vibrant dans ma gorge

un désir cherchant un corps

pour le rendre réel

dans la mémoire d'un mois de novembre

vidé de regards et de lèvres ${ }^{12}$

Le sentiment très fort d'étrangeté à la langue qui se manifeste dans la poésie acadienne lui donne une tonalité tout à fait actuelle dans les meilleurs des cas, mais peut aussi la conduire à la platitude d'une prose disposée en vers sans musique et sans art, mais rarement sans âme et ces carences mêmes en accentuent parfois étrangement l'émotion.

La sortie de la poésie acadienne du nationalisme régionaliste me semble attestée par la diversité dont elle fait preuve. Le meilleur exemple en est Serge Patrice Thibodeau dont la montée en flèche constitue un phénomène inouï en littérature québécoise récente. Quand a-t-on vu un jeune écrivain rafler six prix littéraires à ses six premiers livres, dont le prestigieux prix du Gouverneur général? Voilà pourtant un poète tout à fait atypique par rapport aux stéréotypes du poète acadien. À "l'écriture timide qui n'emplit

11 Jean-Michel Rabaté, "La poésie entre quête et désenchantement ", Le grand atlas des littératures, Encyclopaedia Universalis, 1990, p. 257.

12 Fredric Gary Comeau, Trajets, Moncton, Éditions Perce-Neige, 1996, p. 41. 
14

pas sa ligne" comme l'écrit ici-même Alain Masson, il oppose la profusion de longs vers, de longs poèmes et de longs recueils publiés à un rythme époustouflant, écrits dans une langue précieuse et recherchée, qui arrive à dire de manière poétique aussi bien l'érotisme le plus explicite que le mysticisme le plus exalté. Voilà une poésie des plus exportables, nettoyée de presque tout régionalisme, nourrie de la rencontre des cultures à l'échelle planétaire et s'appuyant sur une réception enthousiaste dans son lieu d'origine. Mais sans doute pour la poésie acadienne s'agit-il de l'exception qui confirme la règle. De plus, pour l'institution littéraire québécoise, ce poète est déjà plus québécois qu'acadien puisqu'on le présente conme "poète québécois d'origine acadienne " ${ }^{13}$, formule dont la lourdeur trahit bien l'embarras et qui, en le rattachant à deux terroirs, sied fort mal à ce globe-trotter qui préférerait sans doute se faire appeler tout simplement "poète".

L'actualité de la poésie acadienne, c'est aussi son plurilinguisme naturel, d'autant plus que la majorité de ses poètes viennent du sud-est du Nouveau-Brunswick ou l'habitent maintenant, regroupés autour de ce que Claude Beausoleil a baptisé "l'école de Moncton". L'anglais et le chiac laissent de plus en plus de traces dans la poésie acadienne et comme pour l'étrangeté à la langue et l'exclusion, il faut faire la part des choses entre ceux qui savent tirer un parti créateur de ce mélange des langues et ceux qui simplement le subissent ou croient qu'il est en lui-même le gage de la création. Il faut ici distinguer entre le défaut de langue et la langue en défaut. Le premier résulte d'un manque de maîtrise de la langue et il ne donne jamais accès par lui-même à la littérature; la seconde est au-delà de la maîtrise de la langue, le retournement de celle-ci contre ellemême, la mise en évidence de ses lacunes qui est au coeur de l'entreprise littéraire de notre temps. Le premier révèle les défauts d'une parole, la seconde les défauts de la langue. La littérature acadienne oscille entre l'un et l'autre, parfois dans le même texte.

\section{Et enfin le roman}

Il faut quand même en venir au roman, qu'on n'osera pas qualifier de parent pauvre, lui qui a offert à la littérature québé-

13 Dans un enregistrement diffusé à l'émission Dimanche-magazine de la radio de Radio-Canada, c'est ainsi qu'on le présentait au Festival de poésie de Trois-Rivières. 
coise son seul prix Goncourt! C'est un fait qu'Antonine Maillet occupe ici une très grande place. Son oeuvre construit ce formidable paradoxe de représenter un peuple dominé, et que de toutes parts on dit dominé dans sa parole, silencieux, honteux de sa langue, en mettant en scène une grandiose célébration de la parole pleine, hyperbolique, joyeuse et jouisseuse, en un mot rabelaisienne. Ce n'est pas pour rien que ça s'appelle de la fiction, mais le pari est tenu et cet univers de comparses acadiens "aussi pittoresques et gueulards que tous les héros de Balzac ou de Zola..." ${ }^{14}$ efface toute trace du "silence qui a duré cent ans"15. Aux Acadiens qui se demanderaient si le français qu'ils parlent est encore du français, Antonine Maillet propose des Acadiens dont le français est plus français que le français de France parce qu'il a miraculeusement conservé la pureté des origines. Elle aura peu d'imitateurs qui la suivront sur ce terrain de la valorisation à l'extrême de la langue ancienne, mais elle en aura beaucoup qui comme elle feront de l'histoire la seconde nature du roman. De la déportation, en passant par l'empremier, où qu'il se situe, jusqu'au début du siècle ou même aux années cinquante, le roman acadien raconte surtout le passé. Ceux qui voudraient figer l'Acadie dans la vision d'une petite société, isolée et archaique, ayant raté le virage industriel et la laïcisation, comme hors du monde et du temps y trouveront toutes les confirmations qu'ils recherchent.

Mais il existe quelques exceptions remarquables à cette tendance qu'il est d'autant plus important de souligner. France Daigle représente l'exact contre-pied d'Antonine Maillet. Elle s'inscrit d'emblée dans la lignée des métafictions modernes où la littérature devient son propre sujet et où le jeu du texte n'exclut pas le matériau culturel acadien mais, selon l'opposition saussurienne bien connue entre langue et parole, le fait régresser de parole constituée à l'état de langue comme réservoir où l'on puisera les éléments qui entrent dans le jeu de la création. En ce sens les romans de France Daigle sont tout à fait typiques de la fiction moderne qui efface la frontière entre l'écriture et la vie, joue des ambiguiités produites par une écriture néanmoins précise et ne renonce pas à la représentation. Le rapport à la langue dans les romans de France Daigle est aussi l'exact opposé de la célébration mailletienne de la parole. Laconisme, litote, ellipse et silence

14 Antonine Maillet, Le chemin Saint-Jacques, Montréal, Léméac, 1996, p. 276.

15 Ibid., p. 337. 
16

forment l'armature du discours narratif de France Daigle, à ses débuts du moins et ce n'est que dans ces livres plus récents que la méfiance fondamentale envers le langage a pris des tournures plus fluides, mais non moins contrôlées.

Jean Babineau, bien qu'il n'ait publié qu'un seul roman alors que France Daigle en compte huit, mérite aussi le détour. Son Bloupe (1993) est la première véritable mise en scène du foisonnement linguistique monctonien. L'alternance déjà audacieuse entre le français, l'anglais et le chiac n'est que le premier pas dans cette entreprise de trituration des discours, des mots et des idiomes. Inscrit sous le patronage de Joyce, le roman met en scène un personnage éponyme avatar de Bloom et il accueille volontiers le calembour bilingue: "... Acadye (Like in the verb dying) (to die)...(p. 172). Le tout se déroule dans une atmosphère de parodie délirante qui tourne en rond beaucoup plus qu'elle ne progresse et qui, toute proportion gardée, rappelle, par la pratique de l'autodérision et de la vraie-fausse candeur comme modes de survie et de perdition, certains accents de Réjean Ducharme. On peut certes voir dans ce roman une représentation émouvante du dilemme linguistique de l'écrivain acadien du sudest : avec une langue en moins et trois langues en trop, il est partagé entre quatre langues impossibles: l'acadien traditionnel et mythique, qui n'est la langue de personne sauf dans les romans d'Antonine Maillet, la langue française idéale, élitiste et inaccessible, la langue anglaise, langue du pouvoir et de l'emprise sur le monde, mais néanmoins pour l'Acadien la langue de l'Autre malgré sa proximité, et finalement le chiac dont l'existence même atteste de l'impossibilité des trois autres - on ne crée pas une langue pour rien - langue stigmatisée et interdite. Pour tous, le français reste la langue principale de l'écriture, mais, pour le meilleur et pour le pire, avec quels tiraillements et distorsions.

Alain Masson a parlé avec raison du "déficit romanesque acadien" ${ }^{16}$ : sauf quelques exceptions, certes importantes, le roman n'offre pas le même intérêt que la poésie, beaucoup plus originale et inventive. Mais il se pourrait que ce déficit commence à se résorber. Dans son dernier roman, Pas pire, qui paraît en avril 1998, France Daigle atteint au sommet de son art. Gérald Leblanc publie en février 1998 son premier roman, Moncton Mantra, qui

16 Alain Masson, "Une certaine iclée de la littérature", Revue de l'Université de Moncton, vol. XXX, $\mathrm{n}^{\circ} 1$, automne 1997. 
n'a rien de traditionnel et qui à sa manière sans doute moins ludique mais non moins excessive retrouve l'univers de Bloupe de Jean Babineau. Enfin, Herménégilde Chiasson et Roméo Savoie sont en train d'écrire des romans...

Même 25 ans après sa renaissance, la littérature acadienne reste une littérature d'instinct: ses auteurs n'ont pas réussi à produire une réflexion sur leur pratique d'écriture qui pourrait éclairer les alternatives qui s'offrent à eux et mesurer la portée de leurs choix. Herménégilde Chiasson a assuré presque à lui seul jusqu'ici ce rôle de conscience de la littérature acadienne et on pourra juger ici-même avec quelle perspicacité dans un texte où il ne manque pas de lancer quelques avertissements à ses compagnes et compagnons de route. L'absence de métatexte donne parfois à la littérature acadienne des allures désinvoltes; on l'imaginerait plus grave, étant donné la précarité qui l'entoure. Cependant, ce qu'elle perd par manque de retour sur elle-même, la littérature acadienne le gagne en audace et en spontanéité. Je ne partage pas le point de vue de Chiasson lorsqu'il avance icimême la familiarité des nouveaux écrivains avec Derrida; je les crois au contraire et comme leurs aînés beaucoup plus près de l'autodidacte que de l'intellectuel patenté. Pour son plus grand bien, la littérature acadienne est tout le contraire d'une littérature écrite par des professeurs, ce que deviennent parfois les littératures minoritaires par simple appel du vide. Si les auteurs acadiens ont quelque chose d'exemplaire, c'est que, tout en étant conscients de s'adresser à un public extrêmement restreint, plusieurs d'entre eux ont choisi de faire de l'écriture leur activité dominante, qu'ils combinent parfois avec une autre forme d'art comme le cinéma, la peinture ou la sculpture. Que Gérald Leblanc, Herménégilde Chiasson, Roméo Savoie, Rose Després, Dyane Léger, France Daigle, Daniel Dugas puissent vivre, non sans difficultés certes, de leur art ou pour leur art dans un si petit milieu est un très fort témoignage qu'ils tiennent l'écriture comme une vocation et non comme un hobby du dimanche.

Il semble que le destin des littératures de l'exiguité soit d'essayer d'en sortir ${ }^{17}$. L'Acadie quant à elle ne sortira pas des conditions objectives de son exiguité, mais sa littérature ne correspond déjà plus aux stéréotypes des littératures mineures. La

17 Voir le dossier * Littératures francophones hors-Québec: lutter contre l'exiguïté., Lettres québécoises, $\mathrm{n}^{\circ} 88$, hiver 1997, p. 7-9. 
littérature acadienne ne sera jamais une littérature hégémonique; elle n'en a pas les moyens et on s'en réjouit, mais cela n'exclut pas qu'elle puisse produire quelques grands écrivains. C'est avant tout ce qu'elle représente par rapport aux Acadiens qui justifie son existence : elle leur permet d'exprimer leur rapport au monde et de se connaître et se reconnaitre dans ces univers fictifs. Mais on sait que pour que cette fonction soit remplie avec toute son efficacité, il lui faut aussi une reconnaissance extérieure qui en confirme la légitimité. Sur ces deux plans, aussi bien intérieur qu'extérieur, la littérature acadienne a encore beaucoup de chemin à faire, car l'exiguité, c'est d'abord au niveau du lectorat qu'elle se fait sentir. Dans ce sens, ce dossier sur l'actualité de la littérature acadienne présenté dans les pages d'une revue québécoise comme Tangence est à marquer d'une pierre blanche. 